\title{
Polariton Electric-Field Sensor
}

\section{Journal Article}

\section{Author(s):}

Togan, Emre; Li, Yufan; Faelt, Stefan; Wegscheider, Werner; Imamoglu, Atac

Publication date:

2020-08-07

\section{Permanent link:}

https://doi.org/10.3929/ethz-b-000430891

Rights / license:

In Copyright - Non-Commercial Use Permitted

Originally published in:

Physical Review Letters 125(6), https://doi.org/10.1103/physrevlett.125.067402

Funding acknowledgement:

671000 - Interacting polaritons in two-dimensional electron systems (EC) 


\title{
Polariton Electric-Field Sensor
}

\author{
Emre Togan $\odot,{ }^{1}$ Yufan Li, ${ }^{1}$ Stefan Faelt, ${ }^{1,2}$ Werner Wegscheider, ${ }^{2}$ and Atac Imamoglu ${ }^{1}$ \\ ${ }_{1}^{1}$ Institute of Quantum Electronics, ETH Zurich, CH-8093 Zurich, Switzerland \\ ${ }^{2}$ Solid State Physics Laboratory, ETH Zurich, CH-8093 Zurich, Switzerland
}

(Received 7 April 2020; revised 2 June 2020; accepted 8 July 2020; published 5 August 2020)

\begin{abstract}
We experimentally demonstrate a dipolar polariton based electric-field sensor. We tune and optimize the sensitivity of the sensor by varying the dipole moment of polaritons. We show polariton interactions play an important role in determining the conditions for optimal electric-field sensing, and achieve a sensitivity of $0.12 \mathrm{~V} \mathrm{~m}^{-1} \mathrm{~Hz}^{-0.5}$. Finally, we apply the sensor to illustrate that excitation of polaritons modifies the electric field in a spatial region much larger than the optical excitation spot.
\end{abstract}

DOI: 10.1103/PhysRevLett.125.067402

Because of their interacting nature, microcavity polaritons have been extensively researched in the context of quantum fluids of light [1]. Polaritons have also been utilized to demonstrate novel optoelectronic devices such as parametric amplifiers [2], all optical transistors [3,4], resonant tunneling diodes [5], and all optical phase shifters [6]. In an alternate application, we demonstrate that polaritons with a large permanent electric dipole moment (dipolar polaritons) are also sensitive electric-field detectors. The detection principle relies on changes in the reflected optical power of a resonant laser upon the application of an electric field, which changes the transition energy of the dipolar polaritons. The resulting sensor, in principle, is fast, responds to changes in the electric field within the polariton lifetime, its sensitivity is tunable by changing the dipole moment of polaritons, and its ultimate performance is determined by the interactions among polaritons. Such a sensor is suited to optical detection of local electric fields that are within the sample. These electric fields could be created by other optically accessible excitations (e.g., indirect excitons $[7,8]$ ) or could be due to excitations that are optically inaccessible (e.g., dark excitons [9] and electric charges). Detection of such electric fields will lead to a clearer understanding of the effects of optically induced environment on polaritons $[10,11]$. The sensitivities that we achieve are sufficient to detect such particles far from the optical probing spot.

We realize polaritons that have an electric dipole moment by nonperturbatively coupling cavity photons with direct excitons (DX) and indirect excitons (IX) in an $\mathrm{In}_{0.04} \mathrm{Ga}_{0.96} \mathrm{As}$ coupled quantum well (QW) structure [12,13]. Electron tunneling between the two QWs facilitates coherent coupling between IX and DX states. By embedding such structures in a planar cavity dipolar polaritons are formed. We focus on the lowest energy polaritons (lower polariton, LP) for sensing, and thus take advantage of the large electric dipole of indirect excitons (dipole length $d \sim 29 \mathrm{~nm}$ ) as well as the narrow linewidth $(\Gamma \sim 50 \mu \mathrm{eV}, \mathrm{FWHM})$ and the high optical reflection contrast of cavity photons. The change of the net growth direction electric field $(\Delta E)$ at the polariton location leads to a change in the polariton transition energy $\sim e d \Delta E$, where $e$ is the electron charge, which in turn changes the reflected power of a laser that is nearly resonant with the polariton transition. We use this simple concept to realize the polariton electric-field sensor.

The sample structure used in this Letter is illustrated in Fig. 1(a). The coupled QW structure resides in a $p-i-n$ diode, and we bias the diode with a dc potential $\left(V_{\mathrm{dc}}\right)$ as well as a small ac potential $\left(V_{\mathrm{ac}}\right)$. The diode bias changes the relative detunings between the different exciton levels, hence with $V_{\mathrm{dc}}$ we can tune the dipole moment, $e d$, of the polariton resonances. Figure 1(c) illustrates this for the LP, where the dipole length of the LP transition is tuned from $d=0 \mathrm{~nm}$ at $V_{\mathrm{dc}}=9.9 \mathrm{~V}$ to $d=2 \mathrm{~nm}$ at $V_{\mathrm{dc}}=8.5 \mathrm{~V}$. We use a sinusoidal ac potential to create a field to be sensed $\left(E_{\mathrm{ac}}\right)$. The resulting controlled sublinewidth $\left(e d E_{\mathrm{ac}} \ll \Gamma\right)$ oscillating electric field is used to characterize performance and limits of the polariton electric-field sensor. The oscillating field allows us to reduce the effects of low frequency noise that is present in our setup. A plot of the amplitude of the change in the reflected optical power at the modulation frequency (that we call $\Delta R$ ) as a function of laser frequency is shown in Fig. 1(d). As expected, $\Delta R$ signal is maximal at the points where the reflection signal has the highest derivative with respect to the laser frequency. We next characterize the sensitivity of the detector.

The sensitivity $(\eta)$ is defined as the minimum detectable field that yields a signal-to-noise ratio of unity for $1 \mathrm{~s}$ of integration [14]. To estimate the sensitivity we record a time trace of $\Delta R$ at each laser detuning for a duration of $1 \mathrm{~ms}$. This $1 \mathrm{~ms}$ interval is broken into subintervals of length $\tau=50 \mu$ s (integration time). We determine $\Delta R$ for each of these subintervals and record the value. We then calculate the standard deviation of $\Delta R$ within the $1 \mathrm{~ms}$ duration which gives us a signal-to-noise ratio for $\tau=50 \mu \mathrm{s}$. We use the fact that the standard deviation of 


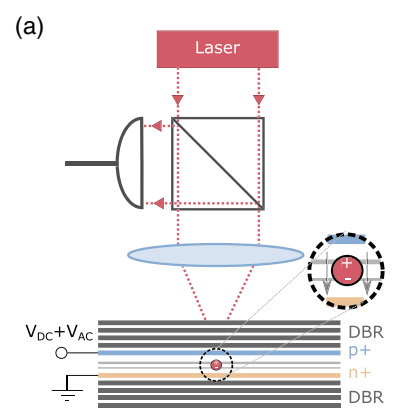

(c)

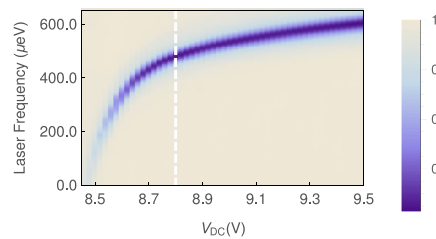

(b)

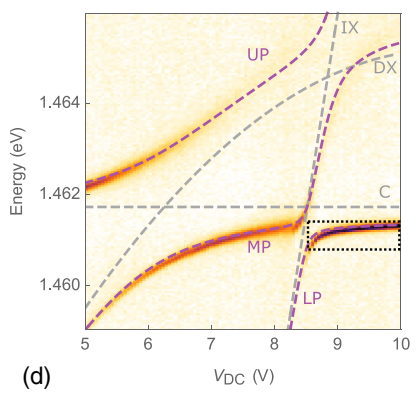

(d)

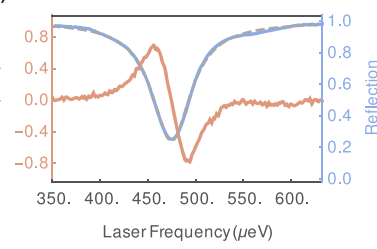

FIG. 1. (a) Schematic of the experimental setup and the sample structure. Sample is held at $\sim 10 \mathrm{~K}$ inside a flow cryostat. The coupled QW structure is located at an antinode of a planar cavity that is formed between two distributed Bragg reflectors (DBRs). We treat polaritons within the $10 \mu \mathrm{m}$ optical excitation spot as a single mode. (b) White light reflection spectrum as a function of $V_{\mathrm{dc}}$. Three polariton branches: lower (LP), middle (MP), and upper (UP) are visible in the reflection spectrum (purple lines) and the inferred energies of the cavity mode $(C)$, IX and DX transitions are shown as gray lines. (c) Laser reflection spectrum (200 $\mathrm{nW}$ laser power) for the LP vs $V_{\mathrm{dc}}$. The vertical axis shows the laser frequency; $1.45989 \mathrm{eV}$ shown as $0 \mathrm{eV}$. (d) In blue (right axis), line cut of (c) at $V_{\mathrm{dc}}=8.78 \mathrm{~V}$. In red (left axis) $\Delta R$, amplitude of the reflected power oscillating at the same frequency (76.226 kHz, $2 \mathrm{mV} p-p)$ as the sublinewidth modulation.

$\Delta R$ scales as $\tau^{-1 / 2}$ (see the Supplemental Material [15]) to find the signal-to-noise ratio for $\tau=1 \mathrm{~s}$. We use this inferred signal-to-noise ratio to determine the experimental sensitivity $(\eta)$. The experimental sensitivity for the dataset shown in Fig. 1(c) is illustrated in Fig. 2(c). Figures 2(a) and 2(c) illustrate the maximum $|\Delta R|$ and the best sensitivity occurs at the optimal $V_{\mathrm{dc}}=8.6 \mathrm{~V}$.

To better understand the existence of an optimum $V_{\mathrm{dc}}$ for the sensitivity, we note that for a shot noise limited linear sensor with a Lorentzian line shape the sensitivity is given by (see Supplemental Material [15]):

$$
\eta=\frac{\Gamma \sqrt{1-C / 2}}{\operatorname{Ced} \sqrt{P}},
$$

where $\Gamma$ is the linewidth, $C$ is the reflection contrast, $P$ is the optical power in photons per second, and we assumed a laser-LP detuning of $\pm \Gamma / 2$. All of the parameters in this expression are measured independently for each $V_{\mathrm{dc}}$ and we plot the expected sensitivity (with no additional fit parameters) along with the experimental sensitivity in Fig. 2(c). The two are in close agreement and they both exhibit an optimum sensitivity around $V_{\mathrm{dc}}=8.6 \mathrm{~V}$. The optimum in the sensitivity is due to the trade-off between
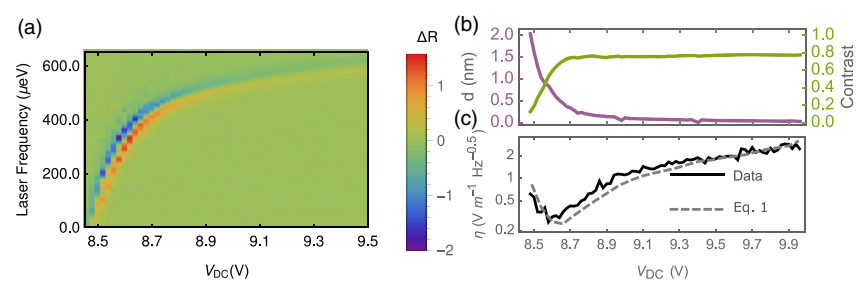

FIG. 2. (a) Plot of $\Delta R$ (arb.units) as a function of laser frequency (1.45989 eV shown as $0 \mathrm{eV}$ ) and $V_{\mathrm{dc}}$ under same conditions as Figs. 1(c) and 1(d). (b) Dipole moment (left axis, purple line) and reflection contrast (right axis, green line) extracted from Fig. 1(c). (c) Experimental sensitivity as a function of $V_{\mathrm{dc}}$. For each value of $V_{\mathrm{dc}}$ we pick the detuning that yields the best sensitivity. To evaluate Eq. (1) we use reflection contrast and dipole moment shown in (b), the linewidth extracted from Fig. 1(c) and detected power $P$ in photons per second.

the increase in the dipole moment and the decrease in contrast as illustrated in Fig. 2(b). The increase in the dipole moment necessitates an increase in the exciton content of polaritons, that introduces nonradiative decay channels $[17,18]$, which reduces the reflection contrast and increases the polariton linewidth. Changes in $V_{\mathrm{dc}}$ also lead to measurable, yet small, changes in the polariton linewidth (see Supplemental Material [15]). Equation (1) predicts better sensitivities than what is measured. This difference is due to the additional noise from the detector, effects of slow variations in the resonance condition (see Supplemental Material [15]), and laser induced electric-field noise. We come back to the last point later in the text. This optimum is the central result of our work and it shows both the dipolar character and high reflection contrast is necessary to achieve good sensitivities. In particular, at the optimum point with $200 \mathrm{nW}$ of incident power, an electric-field sensitivity of $0.26 \mathrm{~V} \mathrm{~m}^{-1} \mathrm{~Hz}^{-0.5}$ is achieved, with an estimated dynamic range of $47 \mathrm{~dB}$ (see Supplemental Material [15]) and an estimated bandwidth that extends to inverse polariton lifetime.

We next explore the effect of incident optical power on our sensor. To better illustrate the effects of higher optical power, we choose a higher modulation frequency (1.750 $181 \mathrm{MHz}$ ), a different gain setting on the photodiode, and $V_{\mathrm{dc}}=9.8 \mathrm{~V}$ with weaker polariton interactions. These changes lead to an initial worse sensitivity for Fig. 3 as compared to Fig. 2. Figure 3(a) shows $|\Delta R|$ as a function of laser frequency for three powers. At higher powers the polariton resonance blueshifts, and the line shape becomes non-Lorentzian $[13,19,20]$. Because of the blueshift, the frequency that yields the best sensitivity changes as a function of power. In addition, at higher powers, an asymmetry in the magnitude of $|\Delta R|$ develops between the blue and red sides of the resonance. As shown in Fig. 3(b), $|\Delta R|$ at the optimal frequency for sensitivity at a given power, increases superlinearly on the blue side, whereas it increases sublinearly on the red side. The green 

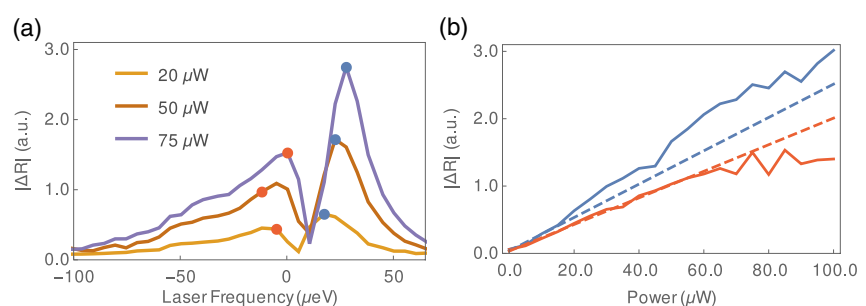

(c)
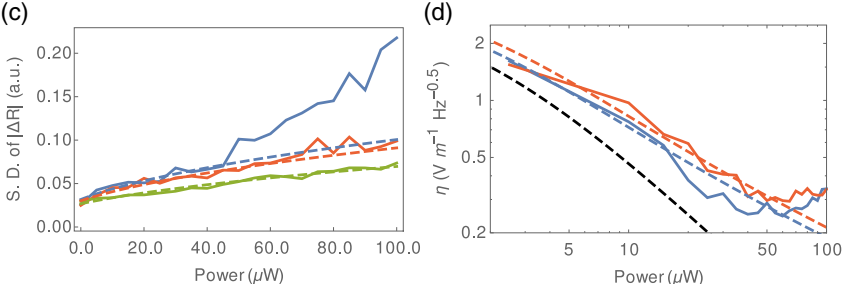

FIG. 3. (a) Change of $|\Delta R|$ vs laser frequency (1.460 $65 \mathrm{eV}$ shown as $0 \mathrm{eV}$ ) for different laser powers. Measurements performed for $1.4 \mathrm{~ms}$ intervals with $\tau=50 \mu \mathrm{s}$. The solid dots indicate the frequency with the best sensitivity at a given power for blue and red detunings. (b) Plot of $|\Delta R|$ as a function of laser power at the laser frequency that yields the best sensitivity for each laser power. Red (blue) lines indicate red (blue) detuning relative to the polariton resonance. Linear fits to data $\leq 20 \mu \mathrm{W}$ are shown as dashed lines. (c) Standard deviation of $\Delta R$ as a function of laser power. The blue and red lines are for the same laser frequencies as in (b). Green line is measured at a relative laser frequency of $-180 \mu \mathrm{eV}$ (far red detuned). The dashed lines are a fit to $\sqrt{A^{2}+B^{2} P}$ the expected standard deviation for detector noise and shot noise. For red and blue curves we fit data points $\leq 40 \mu \mathrm{W}$. (d) Measured sensitivities as a function of laser power. Red and blue solid lines are experimental data measured at the same optimal laser frequencies as in (b). Red and blue dashed lines are the expected sensitivity based on the low power behavior, shown as dashed lines in (b) and (c). Black dashed line is our best-case estimate for detector and shot noise limited sensitivity.

dashed line in Fig. 3(c) illustrates a typical plot of the intensity noise as a function of power in the setup: it is measured as the standard deviation of $\Delta R$ at the laser frequency of $-180 \mu \mathrm{eV}$ (far red detuned). It shows the typical power scaling expected of shot noise with sizable detector noise. The measured noise at laser frequencies that yield the best sensitivities are above this noise level for powers $>1 \mu \mathrm{W}$. At powers higher than $40 \mu \mathrm{W}$, the blue detunings exhibit more noise compared to red detunings.

Combination of these observations imply that the sensitivity of the polariton sensor deviates from the $\propto P^{-0.5}$ power scaling in Eq. (1). The major difference is the saturation of the sensitivity at high powers, observable in Fig. 3(d) for both blue and red detunings above $50 \mu \mathrm{W}$. The second difference is, for blue detunings, the sensitivity is better compared to the prediction of the linear model based on the low power behavior [shown in blue dashed lines in Figs. 3(b)-3(d)] for the power range $20-50 \mu \mathrm{W}$. We do emphasize that the sensor performs poorer compared to a best-case sensitivity estimate shown as the black dashed line. This best-case estimate is calculated with standard deviation estimated from the green dashed curve on Fig. 3(c) and $|\Delta R|$ estimated from the blue dashed curve on Fig. 3(b).

At high powers, such as those used in Fig. 3, polariton interactions lead to a number of rich, nonlinear, phenomena that lead to deviations from the linear model used to derive Eq. (1). The power range used in Fig. 3 is slightly below the threshold for reaching one such phenomenon, optical bistability [19,21]. Within this power range we expect drastic changes in the line shape, as well as significant changes in the intensity noise of the reflected light [20-22]. In terms of electric-field sensing, changes in the line shape lead to the superlinear (and sublinear) increases of $|\Delta R|$ with power, and increased fluctuations in intensity of the detected light lead to increase in the standard deviation of $\Delta R$ and the eventual saturation of sensitivity with power. As interactions are the underlying cause of the above effects, interactions limit the power range that can be utilized with polaritons, hence the sensitivity that can be achieved. In the results presented in Fig. 3 there is significant noise in excess of the fundamental noise limit imposed by polariton interactions. We next turn our attention to one cause of the excess noise: laser induced electric noise that is ubiquitous in structures such as our sample [13,23].

We utilize the polariton sensor to measure a spatial dependence of the laser induced electric noise as well as its time response. As illustrated in Fig. 4(a) we pick two spots on our sample, each $\sim 10 \mu \mathrm{m}$ in diameter, that are separated by $l$. Polaritons on one spot (probe) are used as electric-field sensors. We vary conditions for exciting polaritons at a second (pump) spot and record changes in the electric field (at probe) as a function of these conditions. Pumpprobe experiments are carried out with $V_{\mathrm{ac}}=0 \mathrm{~V}$ and $V_{\mathrm{dc}}=8.8 \mathrm{~V}$. On the probe spot, we excite polaritons with $200 \mathrm{nW}$ of incident power and at a laser detuning of $+\Gamma / 2$. In a first experiment, we pick $l=176 \mu \mathrm{m}$, and we record changes of the electric field at the probe as we vary the photon frequency of the intensity modulated pump laser. Changes in the electric field at probe are detected at the pump modulation frequency. Figure 4(b) shows three detected resonances that correspond to excitation of the three polariton branches at the pump. Of the three branches, the MP has the largest cavity content and narrowest linewidth. Consequently driving this resonance leads to excitation of the largest number of polaritons, as well as the largest detected electric field. In a second experiment we focus on the excitation of MP at different pump spots. At each location we sweep the laser photon frequency to excite the MP and record the maximum detected electric field at the probe. As shown in Fig. 4(c) the detected electric field shows remarkably little change as $l$ changes from 140 to $220 \mu \mathrm{m}$.

This spatial dependence is consistent with an electric field created by the pump induced photocurrent and 


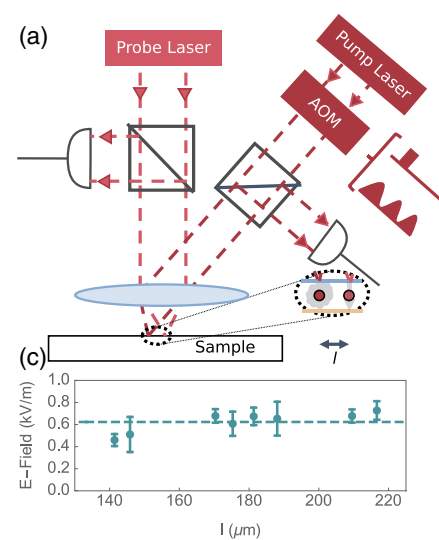

(b)

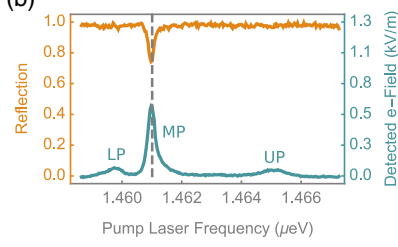

(d)

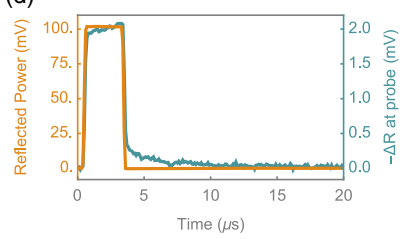

FIG. 4. (a) Schematic of the setup used in the pump-probe experiments. The probe laser and pump laser are focused on two separate spots using a galvo mirror. An AOM is used to modulate the pump laser's power either in sinusoidal waveform (at $76.226 \mathrm{kHz})$ or in a rectangular $(3 \mu \mathrm{s})$ pulse. The reflection at both the probe and pump spots is measured simultaneously. (b) Reflection at the pump spot (yellow, left axis) and detected electric field at the probe spot (cyan, right axis) for $l=176 \mu \mathrm{m}$ as a function of pump laser frequency. Mean pump power is $500 \mathrm{nW}$. Dashed vertical line shows the energy used in (d). (c) A plot of the maximum electric field detected at the probe as the pump spot location is varied. We group points that are within $10 \mu \mathrm{m}$ into a single point and plot their mean as the data point and their standard deviation as error bars vs $l$. (d) Time trace of the reflected power at the pump spot (yellow, left axis) and detected electric field (cyan, right axis) at $l=176 \mu \mathrm{m}$ for a pulsed pump laser.

associated changes in the electric potential on the $p$ and $n$ doped layers (see the Supplemental Material [15]). Such a peculiar spatial dependence of the electric field rules out the possibility that the field is created by localized dipoles (e.g., polaritons) or localized charges (e.g., due to occasional ionization of polaritons [13]).

Photocurrent induced changes in the potential should be accompanied by finite rise and fall times due to the capacitance that is present in the sample. In a third experiment we excite polaritons using a short pulse and measure the changes in the detected electric field as a function of time. As Fig. 4(c) demonstrates the sensed electric field exhibits rise and fall times within $\mu$ s timescales, which is much longer compared to the polariton lifetime.

These experiments highlight a mechanism in which excitation of polaritons create changes in its electrical environment that extends hundreds of micrometers and that persists for microseconds. Our findings are consistent with the mechanism being caused by photocurrent that is induced at the pump location. Such a mechanism not only acts as a noise for high power sensing measurements limiting the sensitivity achievable, but could also alter polariton dynamics in nontrivial ways. We expect the influence of this mechanism could be mitigated using pump-probe experiments that probe dynamics on polariton lifetimes, and hence complete before the capacitance of the sample is charged. To alleviate the effects in continuous wave experiments, different geometries to reduce the resistivities of the conductive layers could be used as well as heterostructure engineering to reduce the photocurrent.

Our experiments demonstrate that polaritons are useful electric-field sensors. We used a sensor diameter of $10 \mu \mathrm{m}$ that is suitable for high resolution spatially resolved electric-field detection. The spatial resolution may be improved to $2 \mu \mathrm{m}$ using state of the art fiber cavities $[17,18]$. The sensitivities that we have demonstrated $0.26 \mathrm{~V} \mathrm{~m}^{-1} \mathrm{~Hz}^{-0.5}$ in Fig. 2 and $0.12 \mathrm{~V} \mathrm{~m}^{-1} \mathrm{~Hz}^{-0.5}$ shown in Supplemental Material [15] are an order of magnitude better compared to similarly sized solid state optical electric-field sensors such as room temperature nitrogen vacancy center ensembles $\left(10 \mathrm{~V} \mathrm{~m}^{-1} \mathrm{~Hz}^{-0.5}\right)$ [24], or cryogenic single quantum dot based electric-field sensors $\left(1-5 \mathrm{~V} \mathrm{~m}^{-1} \mathrm{~Hz}^{-0.5}\right)$ [25-27]. The sensitivity is 2 orders of magnitude worse compared to state of the art electricfield sensors such as single electron transistors [28,29] and electromechanical resonators [30,31] and is 4 orders of magnitude worse compared to millimeter scale conventional electric-field sensors that rely on the electro-optic effect $[32,33]$. The fundamental sensitivity achievable by polariton sensors can be improved, for example, by using state of the art DBR cavities, by using larger sensing areas and higher optical powers that then reduce the effective nonlinearity in the system. One other avenue to improve sensitivity is to take advantage of quantum effects observed with polaritons $[17,18,21,22]$ to realize quantum enhanced polariton sensors.

As demonstrated in this Letter, such polariton based electric-field sensors are particularly suited to detecting charge or electric-field distributions that are within the $p-i-n$ diode structure, such as the presence of remote dipolar polaritons. With the sensitivity demonstrated in this Letter, the electric field created by $\sim 1500,2 \mathrm{~nm}$ dipoles $15 \mu \mathrm{m}$ away should be detectable in $1 \mathrm{~s}$. This opens up possibilities for nondestructive detection of polaritons, for example in pump-probe experiments, and can be used as probes for the intracavity squeezing of polaritons. Polariton, or dipolar exciton, based sensing layers may be embedded during fabrication in close proximity to detect nontrivial charge distributions in 2DEGs or van der Waals materials [34-36]. Such optical measurements allow for probing square micrometer scale areas with good spatial resolution that are inaccessible with global electrical measurements. Moreover, the polaritons are sensitive to magnetic fields due to the diamagnetic and Zeeman shifts [37,38], and based on our results we estimate magnetic field sensitivities on the order of $100 \mathrm{nT} \mathrm{Hz}^{-0.5}$ with $1 \mu \mathrm{W}$ of incident power are achievable.

The data that support the findings of this Letter are available in the ETH Research Collection [39]. 
The Authors acknowledge insightful discussions with Hyang-Tag Lim and Young-Wook Cho. This work is supported by NCCR QSIT and an ERC Advanced investigator grant under the Grant Agreement No. 671000 (POLTDES).

[1] I. Carusotto and C. Ciuti, Rev. Mod. Phys. 85, 299 (2013).

[2] P. G. Savvidis, J. J. Baumberg, R. M. Stevenson, M. S. Skolnick, D. M. Whittaker, and J. S. Roberts, Phys. Rev. Lett. 84, 1547 (2000).

[3] D. Ballarini, M. De Giorgi, E. Cancellieri, R. Houdré, E. Giacobino, R. Cingolani, A. Bramati, G. Gigli, and D. Sanvitto, Nat. Commun. 4, 1778 (2013).

[4] T. Gao, P. S. Eldridge, T. C. H. Liew, S. I. Tsintzos, G. Stavrinidis, G. Deligeorgis, Z. Hatzopoulos, and P. G. Savvidis, Phys. Rev. B 85, 235102 (2012).

[5] H. S. Nguyen, D. Vishnevsky, C. Sturm, D. Tanese, D. Solnyshkov, E. Galopin, A. Lemaître, I. Sagnes, A. Amo, G. Malpuech, and J. Bloch, Phys. Rev. Lett. 110, 236601 (2013).

[6] C. Sturm, D. Tanese, H. S. Nguyen, H. Flayac, E. Galopin, A. Lemaître, I. Sagnes, D. Solnyshkov, A. Amo, G. Malpuech, and J. Bloch, Nat. Commun. 5, 3278 (2014).

[7] M. Stern, V. Umansky, and I. Bar-Joseph, Science 343, 55 (2014).

[8] A. A. High, J. R. Leonard, A. T. Hammack, M. M. Fogler, L. V. Butov, A. V. Kavokin, K. L. Campman, and A.C. Gossard, Nature (London) 483, 584 (2012).

[9] Y. Mazuz-Harpaz, K. Cohen, M. Leveson, K. West, L. Pfeiffer, M. Khodas, and R. Rapaport, Proc. Natl. Acad. Sci. U.S.A. 116, 18328 (2019).

[10] Y. Sun, Y. Yoon, M. Steger, G. Liu, L. N. Pfeiffer, K. West, D. W. Snoke, and K. A. Nelson, Nat. Phys. 13, 870 (2017).

[11] M. Pieczarka, M. Boozarjmehr, E. Estrecho, Y. Yoon, M. Steger, K. West, L. N. Pfeiffer, K. A. Nelson, D. W. Snoke, A. G. Truscott, and E. A. Ostrovskaya, Phys. Rev. B 100, 085301 (2019).

[12] P. Cristofolini, G. Christmann, S. I. Tsintzos, G. Deligeorgis, G. Konstantinidis, Z. Hatzopoulos, P. G. Savvidis, and J. J. Baumberg, Science 336, 704 (2012).

[13] E. Togan, H.-T. Lim, S. Faelt, W. Wegscheider, and A. Imamoglu, Phys. Rev. Lett. 121, 227402 (2018).

[14] C. L. Degen, F. Reinhard, and P. Cappellaro, Rev. Mod. Phys. 89, 035002 (2017).

[15] See Supplemental Material at http://link.aps.org/ supplemental/10.1103/PhysRevLett.125.067402 for details on the measurement setup and the sample structure, measurements of changes in the measured linewidth with $V_{\mathrm{dc}}$, an estimate of dynamic range of the sensor, a description of influence of additional noise sources on sensitivity, an estimate of change in electric potential due to photocurrent, and measurements of the best electric-field sensitivity; Supplemental Material includes Refs. [13,16]

[16] H. Deng, H. Haug, and Y. Yamamoto, Rev. Mod. Phys. 82, 1489 (2010).

[17] A. Delteil, T. Fink, A. Schade, S. Höfling, C. Schneider, and A. İmamoğlu, Nat. Mater. 18, 219 (2019).
[18] G. Muñoz-Matutano, A. Wood, M. Johnsson, X. Vidal, B. Q. Baragiola, A. Reinhard, A. Lemaître, J. Bloch, A. Amo, G. Nogues, B. Besga, M. Richard, and T. Volz, Nat. Mater. 18, 213 (2019).

[19] A. Baas, J. P. Karr, H. Eleuch, and E. Giacobino, Phys. Rev. A 69, 023809 (2004)

[20] T. Fink, A. Schade, S. Höfling, C. Schneider, and A. Imamoglu, Nat. Phys. 14, 365 (2018).

[21] T. Boulier, M. Bamba, A. Amo, C. Adrados, A. Lemaitre, E. Galopin, I. Sagnes, J. Bloch, C. Ciuti, E. Giacobino, and A. Bramati, Nat. Commun. 5, 3260 (2014).

[22] J. P. Karr, A. Baas, R. Houdré, and E. Giacobino, Phys. Rev. A 69, 031802(R) (2004).

[23] C. Coulson, G. Christmann, P. Cristofolini, C. Grossmann, J. J. Baumberg, S. I. Tsintzos, G. Konstantinidis, Z. Hatzopoulos, and P. G. Savvidis, Phys. Rev. B 87, 045311 (2013).

[24] J. Michl, J. Steiner, A. Denisenko, A. Bülau, A. Zimmermann, K. Nakamura, H. Sumiya, S. Onoda, P. Neumann, J. Isoya, and J. Wrachtrup, Nano Lett. 19, 4904 (2019).

[25] A. N. Vamivakas, Y. Zhao, S. Fält, A. Badolato, J. M. Taylor, and M. Atatüre, Phys. Rev. Lett. 107, 166802 (2011).

[26] D. Cadeddu, M. Munsch, N. Rossi, J.-M. Gérard, J. Claudon, R. J. Warburton, and M. Poggio, Phys. Rev. Applied 8, 031002 (2017).

[27] C. Arnold, V. Loo, A. Lemaître, I. Sagnes, O. Krebs, P. Voisin, P. Senellart, and L. Lanco, Phys. Rev. X 4, 021004 (2014).

[28] M. J. Yoo, T. A. Fulton, H. F. Hess, R. L. Willett, L. N. Dunkleberger, R. J. Chichester, L. N. Pfeiffer, and K. W. West, Science 276, 579 (1997).

[29] M. H. Devoret and R. J. Schoelkopf, Nature (London) 406, 1039 (2000).

[30] A. N. Cleland and M. L. Roukes, Nature (London) 392, 160 (1998).

[31] J. S. Bunch, A. M. v. d. Zande, S. S. Verbridge, I. W. Frank, D. M. Tanenbaum, J. M. Parpia, H. G. Craighead, and P. L. McEuen, Science 315, 490 (2007).

[32] A. A. Savchenkov, W. Liang, V. S. Ilchenko, E. Dale, E. A. Savchenkova, A. B. Matsko, D. Seidel, and L. Maleki, AIP Adv. 4, 122901 (2014).

[33] V. Calero, M.-A. Suarez, R. Salut, F. Baida, A. Caspar, F. Behague, N. Courjal, L. Galtier, L. Gillette, L. Duvillaret, G. Gaborit, and M.-P. Bernal, Sci. Rep. 9, 8058 (2019).

[34] Y. Shimazaki, I. Schwartz, K. Watanabe, T. Taniguchi, M. Kroner, and A. Imamoğlu, Nature (London) 580, 472 (2020).

[35] Y. Tang, L. Li, T. Li, Y. Xu, S. Liu, K. Barmak, K. Watanabe, T. Taniguchi, A. H. MacDonald, J. Shan, and K. F. Mak, Nature (London) 579, 353 (2020).

[36] E. C. Regan, D. Wang, C. Jin, M. I. B. Utama, B. Gao, X. Wei, S. Zhao, W. Zhao, K. Yumigeta, M. Blei, J. Carlstroem, K. Watanabe, T. Taniguchi, S. Tongay, M. Crommie, A. Zettl, and F. Wang, Nature (London) 579, 359 (2020).

[37] B. Pietka, D. Zygmunt, M. Krol, M. R. Molas, A. A. L. Nicolet, F. Morier-Genoud, J. Szczytko, J. Lusakowski, P. Zieba, I. Tralle, P. Stepnicki, M. Matuszewski, M. Potemski, and B. Deveaud, Phys. Rev. B 91, 075309 (2015).

[38] H.-T. Lim, E. Togan, M. Kroner, J. Miguel-Sanchez, and A. Imamoğlu, Nat. Commun. 8, 14540 (2017).

[39] E. Togan, Y. Li, S. Faelt, W. Wegscheider, and A. Imamoglu, ETH Zurich Research Collection, http://hdl.handle.net/ 20.500.11850/408377 (2020). 